\title{
L'homologie entre l'Homme et les autres Mammifères pour les loci de coloration du pelage
}

\author{
J.J. LAUVERGNE \\ I.N.R.A., Laboratoire de Génétique factorielle \\ Centre de Recherches zootechnique, \\ 78350 Jouy-en-Josas
}

\begin{abstract}
Résumé
Peu de chercheurs se sont intéressés à l'homologie entre l'Homme et les autres Mammifères pour les loci de coloration du pelage bien connus chez ces derniers : $A, B, C, D, E$ et $P$ en particulier.

Ce désintérêt résulte d'un certain masquage de l'action de tels gènes par le fait que l'Homme est alopécique, c'est-à-dire sans pelage où distinguer clairement l'action de mutants aux loci classiques. Le pelage ayant disparu, c'est au niveau de la peau que s'est faite l'évolution colorée pour, dans un premier temps, protéger celle-ci des ardeurs du soleil, principalement des U.V. L'Homme est en effet apparu dans des zones de savanes intertropicales, en altitude. Pour une telle protection mélanique épidermique des variants peu spécialisés et à action faible et cumulative sont, semble-t-il, intervenus, court-circuitant en quelque sorte l'action de mutants majeurs qui auraient pu apparaître aux loci classiques.
\end{abstract}

Par la suite, certaines populations qui migrèrent vers le Nord ont subi une évolution adaptative, de démélanisation du tégument cette fois, car la couche de mélanine, utile sous les basses latitudes, empêchait le passage des ultra-violets devenus plus rares, pour la synthèse de la provitamine $\mathrm{D}$, déclenchant ainsi des rachitismes.

Il résulte de cette évolution une situation complexe et inhabituelle pour une espèce de Mammifères et la formule courante aux loci de base ( $A$ Agouii et E Extension) pour la synthèse alternative de l'eu/et de la phaomélanine ne peut être encore que suggérée : $a a \mathrm{E}^{+} \mathrm{E}^{+}$ou $\mathrm{A}^{\text {? }} \mathrm{A}^{\text {? }} \mathrm{E}^{\mathrm{d}} \mathrm{E}^{\mathrm{d}}$ pour les races fortement mélanisées ainsi que les races mongoliques et la plupart des caucasiens non roux, ces deniers étant $A^{y} A^{v} E^{+} E^{+}$ou aaee ou $A^{?} A^{\text {? }}$ ee.

Les autres loci de coloration qui ont muté n'ont donné semble-t-il que des mutants sans intérêt pour l'adaptation de l'espèce soit aux loci classiques (en $D$, Dilution, avec la phénylcétonurie et en $C$, Albinisme vrai, avec des mutants albinos non encore définitivement localisés) soit à des loci plus rarement signalés chez les Mammifères mais où la variation colorée s'accompagne d'anomalies graves par ex. les Syndromes de Chediak-Higachi ou de Waardenburg. Ces mutants se maintiennent à de faibles fréquences par suite de dérive génétique, d'avantage de l'hétérozygote ou d'effet de fondateur.

L'analyse de la coloration de la peau par des méthodes de génétique quantitative donne des résultats parfois contradictoires qui laissent à penser qu'auprès de polygènes il pourrait exister des gènes à effets plus importants. Il reste à mettre ceux-ci en évidence, ce qui pourrait être possible avec des méthodes histologiques et histochimiques, au moins dans certains cas. 


\section{Introduction}

L'homologie entre espèces pour tout un groupe de loci est la clé de voûte de la génétique de la pigmentation du pelage des Mammifères, spécialement depuis les travaux de Wright $(1917,1918)$ (cf. aussi Haldane, 1927 ; Little, 1958 ; Searle, 1968).

Les méthodes comparées dérivées de cette homologie entre loci ont permis aussi bien la progression des connaissances génétiques spécifiques, que l'élaboration de certains concepts fondamentaux (cf. par exemple la correspondance un gène/un enzyme avancée par WRIGHT dès 1917).

Cette correspondance entre loci d'espèces diverses a été mise en évidence, tout d'abord chez les Rongeurs de laboratoire, puis chez les Carnivores. Actuellement elle s'étend chez les Ruminants (cf. LaUvergne, 1979). Elle est toutefois assez difficile à dégager chez l'Homme si l'on en croit SEARLE (1968 et 1975), qui est après WRIGHT (1918) le principal auteur à s'être préoccupé de cette extension (cf. aussi Spearman, 1977). Cette espèce de Primate vient cependant en tête de toutes les espèces de Mammifères pour le nombre de variants mendéliens décrits (cf. déjà le catalogue de McKusick, 1961, remis à jour périodiquement).

\section{Situation actuelle de l'homologie}

Pour faire le point après SEARLe (1968 et 1975) sur la situation actuelle de l'homologie Homme/Mammifères, nous avons interrogé deux bases bibliographiques automatisées :

- Excerpta medica (Pays-Bas) ;

- Medlars on line (ou Medline) (Etats-Unis), au moyen du terminal du Service de Documentation Centrale de Jouy-en-Josas (C.N.R.Z - I.N.R.A.).

Cette interrogation nous a fourni, après élimination des redondances et des parasites, environ 160 références pertinentes avec leurs résumés pour la période 1975-1980.

Un premier examen de la littérature ainsi accumulée montre qu'au cours de la période considérée, il n'y a pas eu de progrès notable des connaissances en matière d'homologie des loci de coloration entre l'Homme et les autres Mammifères (1). Le locus $D$ (Dilution) reste le seul des loci de base à être identifié à peu près sûrement chez l'Homme avec le mutant de phénylcétonurie, l'imprécision continuant à peser sur le locus $C$ d'Albinisme vrai, auquel il est bien difficile d'attribuer un ou plusieurs des nombreux variants d'albinisme qui continuent régulièrement à être signalés.

(1) Les cinq principaux loci contrôlant la mélanisation et la coloration du pelage chez les Mammifères sont $A$ Agouti, $B$ Brun, $C$ Albinisme, $D$ Dilution, $E$ extension. On peut ajouter $\boldsymbol{P}$ Pink eyed et Ln Leaden qui mutent moins fréquemment (SEarle, 1968; Robinson, 1970). 


\section{Les raisons de la stagnation}

Cet état de stagnation des connaissances homologiques ne semble pas dû uniquement à une désaffection des auteurs pour ce type d'approche comme en témoignerait par exemple le silence de Wasierman (1974). Tous ceux-ci en effet ne manquent pas de rechercher un modèle animal et réussissent parfois à en trouver un, spécialement quand il s'agit d'un syndrome (cf. par ex. JHA, 1975, pour revue).

En fait, bien qu'on note une grande variété de couleurs des téguments et des phanères de l'Homme, ces variations, dont WRIGHT (1918) avait déjà montré le caractère continu, échappent le plus souvent à l'analyse mendélienne avec des gènes majeurs. Les raisons de ce court-circuitage des gènes aux loci majeurs de coloration ont des raisons anatomiques physiologiques qui tiennent à la nature du milieu où l'Homme est apparu et aux migrations postérieures de l'espèce. On peut présenter ces influences et leurs conséquences sur le déterminisme comme suit:

\section{1. - L'Homme est un Primate alopécique qui apparaît dans une zone de savane intertropicale, en altitude}

Pour reprendre l'expression du zoologiste Desmond MorRIs (1968) l'Homme est un singe nu. Cette alopécie n'est pas l'apanage de notre espèce, c'est simplement un moyen de défense contre la chaleur que certaines autres espèces vivant dans le même milieu ont également adopté, ainsi l'Eléphant, le Rhinocéros (cf. RougeOT, 1981).

En zone tropicale de savane d'altitude, le rayonnement solaire intense, spécialement les ultra-violets (U.V.), serait des plus dommageables pour les téguments des êtres alopéciques si ceux-ci ne développaient une pigmentation épidermique mélanique intense, filtre très efficace. C'est le cas des animaux cités et également de l'Homme (Rougeot, 1981).

Il résulte de cette situation tout d'abord une difficulté pour d'éventuels variants aux loci homologues de se manifester, en l'absence même du substrat anatomique (le pelage) qui permet leur réalisation même. En fait, ces variants pourraient se manifester au niveau des téguments mais ils semblent mal adaptés à cette tâche pour des raisons qui en partie nous échappent, mais qui semblent dues au comportement différent des mélanocytes lorsqu'ils colorent l'épiderme et lorsqu'ils fonctionnent dans le follicule (Ortonne \& BenedetTo, 1981). Des facteurs génétiques beaucoup moins spécialisés vont ainsi pouvoir agir pour mélaniser la peau, aux côtés de facteurs environnementaux variés, endogènes et exogènes, court-circuitant, au cours de cette mélanisation du tégument qui a été le lot de notre ancêtre, les variants homologues dont la venue avait pu se faire attendre et dont l'effet n'était plus déterminant. Ainsi s'expliquerait le déterminisme d'aspect quantitatif polygénique de la pigmentation de la peau des races fortement mélanisées que relèvent par exemple PosT \& RAO (1977) et qu'ils analysent au moyen du paramètre d'héritabilité couramment utilisé dans les expériences de sélection pour des caractères quantitatifs, cf. aussi Bonaitı \& LANGANEY (1981). 


\section{2. - Les migrations en latitude induisent une évolution pigmentée secondaire}

Sous les tropiques, vivant de chasse, de cueillette et d'un peu d'agriculture et d'élevage (mais très récemment de ces 2 dernières activités, au plus 10000 ans) les descendants de l'Homme ancien sont tout normalement restés fortement mélanisés. Ainsi s'expliquerait dans l'Ancien Monde la répartition de peuplades fortement mélanisées sous les latitudes intertropicales allant de l'Afrique centrale à la Papouasie Nouvelle Guinée.

Toutefois, lorsque l'Homme a commencé à migrer vers des latitudes plus élevées, spécialement vers le Nord du continent eurasiatique, son environnement s'est modifié avec la diminution régulière de la chaleur ambiante et du rayonnement d'ultra-violets. A ces conditions une forte mélanisation est mal adaptée, à cause en particulier des risques de rachitisme dus au manque de provitamine $\mathrm{D}$ qui a besoin, pour se synthétiser, d'une certaine dose d'ultra-violets que les peaux trop mélanisées arrêtent (Neer, 1975 ; Quevedo et al., 1975 ; Riquet, 1978 ; Post et al., 1975).

Ainsi, en remontant vers le pôle, les populations ont dû, pour survivre, démélaniser leur tégument, ce qui explique le gradient de coloration de la peau retrouvé chez les populations en place depuis longtemps sur un axe allant de l'Afrique centrale au Nord de la Sibérie en passant par le Moyen-Orient.

Comme pour la mélanisation, il est probable que pour la démélanisation sont intervenus pour l'essentiel, au moins au début, des variants autres que les variants à effets majeurs de loci homologues car cette évolution a été progressive, comme le montre l'étalement du gradient sud-nord. C'est ainsi par exemple que dans une première phase de décoloration, on ne note aucune variation des couleurs de la chevelure, qui reste noir de jais.

Par la suite, polygènes et variants à effets majeurs ont pu additionner leur action. Un exemple nous est fourni par les ségrégations spectaculaires des roux dans les populations nordiques cependant que l'on note une gradation insensible du noir de jais au blond clair pour la chevelure avec une association plus ou moins marquée de la coloration de l'iris.

\section{3. - Ultimes complications}

Cette situation, déjà confuse, est encore compliquée semble-t-il par un relâchement récent de la pression de sélection dont les conséquences polymorphiques étaient déjà très visibles dans les zones tempérées (SEARLE, 1968), même sans parler des migrations multiples plus récentes (cf. par exemple la situation de grandes métropoles comme Londres ou New York).

\section{Les possibles interprétations homologiques}

Toutes ces considérations laissent à penser que les progrès pour élucider l'homologie Homme/Mammifères vont être difficiles. On peut cependant esquisser quelques interprétations. 


\section{A. - La situation aux loci Agouti (A) et Extension (E)}

Ces deux loci sont les loci de base pour le contrôle de la répartition combinée de l'eumélanine (noire ou brune) et de la phaeomélanine (rouge) (Prota \& Searle, 1978). L'un ou l'autre, ou les deux de ces loci, ont dû certainement muter puisque l'on n'observe pas de pillosité zonée chez l'Homme (à l'instar des autres Primates et au contraire des singes inférieurs) qui sont données par la formule $A^{+} A^{+} E^{+} E^{+}, A^{+}$ étant l'allèle sauvage parfois appelé agouti, au locus $A$ gouti, et qu'il y a des noirs uniformes et des rouges phaeomélaniques.

La plupart des Hommes, aussi bien nègres que méditerranéens ou même caucasiens blonds, présenteraient le phénotype noir uniforme (uniquement de l'eumélanisme) ce qui voudrait dire qu'ils sont aa $E^{?} E^{?}$ ou $A^{?} A^{?} \cdot E^{\mathrm{d}} E^{\mathrm{d}}$.

L'explication avec $a a$ récessif tiendrait compte de la disparition du phénotype agouti $\left(A^{+}\right.$aurait été remplacé par $a$ ) et de la dominance de certains variants phaeomélaniques (les rouges dominants se trouvent au locus Agouti). Par contre, les rouges récessifs s'expliqueraient mal par le variant $c$ au locus $E$ car, au moins chez la Souris, la combinaison aa/ee donne un pelage noir (LAmoureux \& MEer, 1975). Toutefois, on peut admettre que les espèces ne sont pas entièrement homologues pour les rapports d'épistasie. Ce serait alors l'explication des variants rouges observée en Papouasie Nouvelle Guinée par Nixon (1976).

En faveur de la thèse proposée avec prudence par SEARLE (1975) d'un noir donné par $E^{d}$, il y aurait tout đabord la facile explication des rouges récessifs par $e$ (cf. aussi la discussion de Spearman, 1977). Toutefois, la mutation de $E^{+}$en $E^{d}$ est moins fréquente dans les espèces animales que celle de $A^{+}$en $a$.

Signalons que, si la mise en évidence de poils «agouti»par examen macroscopique des pillosités ne ne trouve pas dans la littérature, cela ne signifie pas qu'ils soient absents chez l'Homme, sous une forme plus difficile à déceler peut-être. Des auteurs comme BREATHNACH et al. (1964) et CÉSARINI (1975) ont en effet mis en évidence la présence simultanée de mélanosomes eu/et phaeomélaniques dans certains mélanocytes. Un examen plus attentif de la pillosité de tels sujets pourrait peut-être permettre de déceler les porteurs d'allèles proches de l'allèle sauvage.

\section{B. - La situation au locus B (Brun)}

l'allèle non muté en $B\left(B^{+}\right)$détermine une eumélanine de couleur noir de jais. Bien qu'aucun variant récessif brun-chocolat n'ai été décrit, SEARLE (1968) pensait qu'il pourrait en exister chez l'Homme.

Dans les populations caucasiennes, leur mise en évidence semble toutefois délicate. Sans preuves histologiques la variation des nuances des cheveux et de la peau est si grande que les éventuels variants en $B$ seraient difficiles à distinguer même pour le cas où l'on saurait la nuance exacte des variants brun-chocolat. On rejoint là, la difficulté rencontrée pour la mise en évidence macroscopique des poils, à la fois eu/et phaeomélaniques.

Une recherche systématique avec une simple échelle colorée pourrait être plus fructueuse parmi les races fortement eumélanisées, à condition de faire la part des 
variations parasites sous l'influence de facteurs non génétiques (rayonnement, alimentation, âge, teinture).

Ces recherches pourraient être complétées par des comparaisons d'images histologiques des pigments comme cela a été fait par ex. chez la Souris (SILVERs, 1980).

\section{C. - La situation au locus $D$}

Le variant de phénylcétonurie a été très étudié chez l'Homme car il est responsable d'une maladie héréditaire grave (cf. par ex. KNOX, 1972). Il est considéré comme l'homologue de $d^{\text {l }}$ (dilute lethal) au locus $D$ de la Souris. Toutefois, outre les différences de détails de comportement relevées déjà par Searle (1968), Silvers (1980), dans une récente revue sur la Souris, relève que l'altération du métabolisme de la phénylalanine demande encore confirmation dans cette espèce. Signalons que le locus de phénylcétonurie a été localisé sur le chromosome $\mathrm{n}^{\circ} 1$ de l'Homme (McKusick, 1980). Récemment, enfin, Griscelli \& Prunieras (1978) pensent avoir trouvé un homologue de l'allèle $d$ (dilute, viable) chez un variant de race blanche. L'homologie semble même plus vraisemblable que dans le cas de l'allèle responsable de la phénylcétonurie.

\section{D. - La situation au locus d'Albinisme vrai $C$}

Elle a été revue par Voulor (1981). On pense que ce locus contrôle la structure de l'enzyme tyrosinase, pièce maîtresse du métabolisme des mélanines. Mais, si la mise en évidence de l'appartenance au locus est facile par test d'allélisme, elle l'est beaucoup moins lorsqu'il faut distinguer l'albinisme total ou subtotal vrai des mimiques, qui sont légions chez l'homme. Des allèles provoquant un affaiblissement de la coloration de l'iris sont peut-être des allèles en $C$ mais leur appartenance à ce locus est pour les raisons vues ci-avant, difficile à mettre en évidence.

\section{E. - Les autres loci homologues}

On a vu plus haut que les variants colorés s'accompagnant d'anomalies avaient été bien étudiés. Ils sont au nombre de quatre au moins chez l'Homme (JAH, 1975) : le syndrome de Tiètze, le syndrome de Waardenburg, le syndrome de Chediak-Higashi et la, déjà citée, Phénylcétonurie. Il s'agit de facteurs homologues avec des variants assez rares dans les autres espèces de Mammifères.

Ces variants accompagnés d'anomalies, de même que les nombreux cas d'albinisme, ne participent pas chez l'Homme à la pigmentation normale des races actuelles. Ce sont des variations parasites, plus ou moins en voie d'élimination ou se maintenant pour des raisons particulières et souvent transitoires (avantage de l'hétérozygote, effet de fondateur dans un groupe isolé, sélection artificielle pour des raisons magiques). La manière dont ils sont présents illustre d'ailleurs le mode particulier, déjà souligné, de la commande génétique de la couleur chez l'Homme : faisant peu appel aux gènes majeurs spécialisés. 


\section{Discussion et conclusion}

Le présent article doit être considéré comme un simple survol du problème de l'extension à l'Homme de l'homologie entre loci de coloration chez les Mammifères car l'extraction des articles pertinents n'est pas exhaustive, le choix des articles cités (que les auteurs non cités nous excusent) peut être discutable, et il y a une part d'interprétation, excusable par la rareté d'ailleurs finalement motivée, des tentatives plus anciennes.

Quoiqu'il en soit, on a pu cependant dégager une bonne partie des raisons pour lesquelles cette homologie est si difficile à mettre en évidence.

De fait, l'évolution de la couleur des phanères et surtout du tégument chez l'Homme, ont été en quelque sorte court-circuité les ressources alléliques qu'auraient pu fournir les principaux loci de coloration, les rendant ainsi silencieux. C'est donc vers ces nouvelles familles de gènes (au pluriel car il pourrait $\mathrm{y}$ avoir les gènes de la mélanisation primaire, puis ceux de la démélanisation secondaire) que doivent donc normalement porter les principaux efforts avec l'aide éventuelle de modèles animaux (par exemple la génétique de la mélanisation de la peau de certaines races ovine, LAUVERGNe et al., 1979). On pourrait ainsi étudier de plus près le chimpanzé à pigmentation cutanée, variable, comme modèle d'eumélanication primaire, cf. PosT et al. (1975).

Avec la revue de BonaITI \& LANGANEy (1981) on voit comment cette apparente multiplicité de gènes à effets mineurs et additifs peut être, avec encore bien des imprécisions dénombrées, et CÉSARINI \& GuÉDON (1981) proposent une voie de mise en évidence de variants par examens histologiques.

Reçu pour publication en mars 1981.

\section{Summary}

\section{The homology between Man and the other Mammals} for the loci of coat coloration

Few research workers have studied the homology between coat color loci in Man and in the other Mammals, although A, B, C, D, E and particularly P, are well known in the latter.

This lack of interest is the result of some masking of the action of such genes because Man is an alopecic animal, that is, he has no hairy coat in which mutant action at the usual loci is clearly distinguishable. Since the coat has disappeared, skin coloration has developed primarily to protect against the radiations of the sun and mainly against U.V. Man has been found in high intertropical savannah zones. Weak, cumulative, not very highly specialized variants of this protection by skin melanin probably occurred, short-circuiting major mutants which might have appeared at the usual loci.

Afterwards, an adaptative change in tegument took place in some populations which had migrated northwards : demelanization. The melanin layer, which had been useful at low altitudes, prevented the U.V., which had become rare, from passing through the skin to activate provitamin D synthesis, and rickets resulted.

This adaptative change presented an unusual, complex situation in a mammalian species, and the present formula, using basic loci ( $A, A g o u t i$ and $E$, Extension) for the alternative synthesis of eu- and phaeomelanin, is still be only indicative : $a a E^{+} E^{+}$or 
$A^{?} A^{?} E^{\mathrm{d}} E^{\mathrm{d}}$ for dark as well as for mongoloid races and most whites which are not red-haired, the latter being $A^{\mathrm{y}} A^{\mathrm{y}} E^{+} E^{+}$or aa ee or $A^{?} A^{\text {? }} e e$.

The other color loci which have mutated do not seem to have produced any mutants to adapt the species either to the usual loci (in D, Dilution, with phenylketonuria, or better delute lethal, and in $C$, True albinism, with albino mutants which have not yet been definitively localized) or to more unusual loci reported in mammals but where color variation is accompanied by serious abnormalities such as Chediak-Higachi disease or Waardenburg syndrome. These mutants are maintained at a low frequency level by genetic drift, heterozygote advantage or the founder effect.

The analysis of skin color by methods of quantitative genetics sometimes gives contradictory results which indicate that near the polygenes may be genes with greater effects. This may be proved, at least in some cases, using histological and histochemical methods.

\section{Références bibliographiques}

Bonatti Catherine, Langaney A., 1981. Génétique formelle des pigmentations humaines à variations continues : beaucoup d'hypothèses, peu de conclusions. Ann. Génét. Sél. anim., 13, (1) 37-42.

Breathnach A.S., Wyllie L.M., 1964. Electron microscopy of melanocytes and melanosomes in freckled human epidermis. J. Invest. Derm., 42, 388-394.

Brues A.M., 1975. Rethinking human pigmentation. Amer. J. Phys. Anthrop., 43, 387-391.

CÉSARINI J.P., 1976. Red-hair, sun-burning skin : melanocyte fine structure. 3rd European Meeting on M.E. applied to Cutaneous Pathology. 2/4/1976.

CÉSARINI J.P., Isabelle GuÉdon, 1981. Possible contrôle de la pigmentation humaine par des gènes à des loci non homologues des loci de coloration classiques des Mammifères. Ann. Génét. Sél. anim., 13, (1) 43-56.

Griscelli C., Prunieras M., 1978. Pigment dilution and immunodeficiency : a new syndrome. Inter. J. Dermato., 17, 788-791.

Haldane J.B.S., 1927. The comparative genetics of colour in Rodents and Carnivora. Biol. Rev., 2, 199-212.

JHA S.K., 1976. Hereditary disorders of cutaneous pigmentation. Ind. J. Pediat., 43, 141-143.

Knox E., 1972. Phenylketonuria, in : J.B. STAndury, J.B. WyngaArden, D.S. Fredrixson, The metabolic Basis of inherited Diseases. Chapter 11, 266-291.

LamoreuX M.L., Mayer T.C., 1975. Site of gene action in the development of hair pigment in recessive yellow (e/e) Mice. Develop. Biol., 46, 160-166.

Lauvergne J.J., 1979. Homology of alleles at the Agouti locus in Ruminants. Ann. Dermatol. Vénéréol. (Paris), 106, 411.

Lauvergne J.J., Cesbron J., Ortonne J.P., Melchior J., Havard C., 1979. Gene or genes for skin pigmentation in the Sheep : a preliminary note. Proc. 2nd Europ. Workshop Mamm. Melanin. Pigment., London, 1979.

Litrle C.C., 1958. Coat color genes in Rodents and Carnivores. Quaterl. Rev. Biol., 33, 103-137.

McKusick V.A., 1961. Mendelian inheritance in man. The Johns Hopkins Press, Baltimore.

McKusick V.A., 1980. The Anatomy of the Human Genome. J. Hered., 71, 370-391.

Morris D., 1968. Le singe nu. Bernard Grasset, Paris.

NeER R.M., 1975. The evolutionary significance of vitamine D, skin pigment and ultraviolet light. Amer. J. Phys. Anthropol., 43, 409-416.

Nixon D.F., 1976. Studies on the mechanism of the inherited Red Skin color found among New Guineans. Pigment Cell, 3, 184-190. 
Ortonne J.P., Benedetto J.P., 1981. Mélanocytes épidermiques et mélanocytes folliculaires. Ann. Génét. Sél. anim., 13, (1) 17-26.

Post P.W., Daniels Jr F., Binford Jr R.T., 1975. Cold injury and the evolution of «White» skin. Human Biol., 47, 65-80.

Post P.W., Rao D.C., 1977. Genetic and environmental determinants of skin color. Am. J. Phys. Anthropol., 47, 399-402.

Post P.W., Szabo G., Keeling M.E., 1975. A quantitative and morphological study of the pigmentary system of the Chimpanzee with the light and electron microscope. Am. J. Phys. Anthrop., 43, 435-444.

Prota G., Searle A., 1978. Biochemical sites of gene action for melanogenesis in Mammals. Ann. Génét. Sél. anim., 10, 1-8.

Quevedo W.C., Fitzpatrick T.B., Pathak M.A., Jimbow K., 1975. Role of light in human skin color variation. Amer. J. Phys. Anthrop., 43, 393-408.

Riquet R., 1981. Valeur adaptative de la pigmentation cutanée. Ann. Génét. Sél. anim., 13, (1) 27-36.

Robinson R., 1970. Homologous mutants in mammalian coat color variation. Symp. Zool. Soc. Lond., 26, 251-269.

Rougeot J., 1981. Déterminisme de la répartition de la pigmentation dans le pelage et la peau des Mammifères. Ann. Génét. Sél. anim., 13, (1) 9-16.

SeARle A.G., 1968. Comparative genetics of coat colour in Mammals. Logos Press Academic Press, New York, London.

SEARle A.G., 1975. Distribution and probable homologies of some genes determining coat color in Mammals, in R.C. KING, Handbook of Genetics, 4, 459-467.

Silvers W.K., 1979. The coat colors of Mice. Springer, New York, Heidelberg, Berlin.

Spearman R.I.C., 1977. The Genetics of Hair Growth and Coloration, in : JarretT, The Physiology and Pathophysiology of the skin, 4. The hair follicule, Academic Press, London, 1458-1494.

Voulot C., 1981. Etude biochimique de l'albinisme chez les Mammifères et chez l'Homme. Ann. Génét. Sél. anim., 13, (1) 51-56.

Wasserman H.P., 1974. Ethnic pigmentation. Exierpta Medica, American Elsevier, Amsterdam and New York.

WRIGHT S, 1917. Coat color inheritance in Mammals. I to VII. J. Hered., 8, 224-235; $373-378$; 426-430 ; 473-475; 476-480 ; 521-527 ; 561-564.

WrIGHT S., 1918. Coat color inheritance in Mammals. VIII Swine to XI. Man. J. Hered., 9, $33-38 ; 87-90 ; 139-144 ; 227-240$. 